\title{
Nutrients Returned from Standing Litter of Four Monocultural And Diverse Land-Use Systems at Brazilian Atlantic Rain Forest: Research Article
}

\author{
Luis Claudio Maranhao Froufe* \\ Brazilian Agricultural Research Corporation, Brazil
}

Submission: May 17, 2019; Published: July 05, 2019

"Corresponding author: Luis Claudio Maranhao Froufe, Brazilian Agricultural Research Corporation, Brasilia, Brazil

\begin{abstract}
The conversion of natural forests into conventional agricultural and cattle systems is very harmful to environmental sustainability. In Brazil, mainly at Atlantic Rain Forest, that deforestation can be observed by a great number of small widespread unconnected fragments, where genetic erosion is a result of reduced above- and belowground biodiversity, increased organic matter decomposition and thus, decreasing soil C storage. Agroforestry systems provide food production, but little is known about how standing litter can affect the nutrient supply for ecological-based agroforestry systems. This work was conducted in family small farms containing conventional agriculture, pasture, ecological-based agroforestry (summarized here as ecological agroforestry) and natural regeneration, where standing litter was collected, separated into different fractions, weighted and analysed for nutrient contents. Standing litter was greater in natural regeneration (13.7 Mgha $\left.{ }^{-1}\right)$ and ecological agroforestry $\left(10.2 \mathrm{Mgha}^{-1}\right)$, than in pasture $\left(4.8 \mathrm{Mgha}^{-1}\right)$ and conventional agriculture $\left(3.6 \mathrm{Mgha}^{-1}\right)$, all mainly composed by leaves and branches. Standing litter of conventional agriculture was richer in N, P and Mg, due to external inputs. Standing litter of ecological agroforestry was richer in Ca, while


$\left(44.3 \mathrm{Mgha}^{-1}\right)$, while ecological agroforestry had greater amounts of $\mathrm{K}\left(346.9 \mathrm{Mgha}^{-1}\right)$. Ecological agroforestry results were similar to natural areas, and significantly higher than monocultures, indicating the importance of litter management for sustainability of diverse land-use systems.

Keywords: Conventional agriculture and pasture; Ecological-based agroforestry system; Multistate agroforestry system; Natural regeneration; Nutrient Additions
\end{abstract}

\section{Introduction}

Ever since in Brazilian history, agricultural policies based on monoculture species cultivated along large areas (e.g. sugar cane, maize, eucalyptus, pasture and/or soybean) had led to severe deforestation of natural forest landscapes, including some of world's biodiversity hotspots. At Atlantic Rain Forest, that deforestation can be observed by a great number of small widespread unconnected fragments; most of them lower than 50ha kilometres away from each other, strongly diversity loss due to edge effects and limited to less than $13 \%$ of its original area $[1,2]$

The conversion of natural forests into conventional agricultural and cattle systems alter soil attributes, specially decreasing soil C storage as a result of lowering the above- and belowground biodiversity and increasing organic matter exposure to decomposition [3-6]. Conversely, plant diversity can influence terrestrial net primary, decomposition and nutrient dynamics through changes in microclimatic conditions, complementary nutrient use and rhizosphere processes [7]. In addition, the amount and quality of plant litter, mainly fast $\mathrm{C}$ pools as leaves and fine roots, have a strong impact on nutrient cycling, $\mathrm{CO}_{2}$ fluxes, soil organic matter formation, nutrient mineralization, and plant growth [8].
The development of an alternative agricultural model, linking food production concurrently to environmental conservation and human social development is, thus, strategically for modern sustainable and profitable agriculture [9].

Agroforestry is a land-use system in which woody perennials species is grown in association with herbaceous plants or livestock, in a spatial arrangement, rotation, or both Young [1997]. Ecological-based agroforestry, also known as multistrata agroforestry systems [10] concept advances toward to natural ecological succession, but with massive human design and management, mainly woody and foliage pruning for water and light/ shade supply, nutrient additions and soil fertility maintenance. Litter production and decomposition are the main factors that enhance agroforestry system sustainability due to massive pruning management [11], but ecological-based agroforestry systems remain an unknown land-use productive/conservative model even with some local practical experiences [12,13], highlighting the importance of how litter and its nutrient additions can affect soil fertility and sustainability in those alternative ecological-based agroforestry systems. 


\section{Methods}

\section{Site description}

This work was conducted at Barra do Turvo, Sao Paulo State, Brazil, characterized by a wavy relief, from30 to 1,000 meters height, and continuous fragments of Atlantic Dense Ombrophyllous Rain Forest [14]. Climate is a subtropical humid mesothermal, with precipitation ranging from 1,500 to $2,000 \mathrm{~mm}$, and $22^{\circ} \mathrm{C}$ mean annual temperature [15].

Family small and medium farms containing different agricultural systems (conventional agriculture, pasture, and ecological-based agroforestry system) and natural forests (natural regenerations) were chosen according to local partners indications and technical criteria. Three replications of each land-use were evaluated. Monoculture agricultural systems were represented by conventional agriculture and pasture, while diverse systems included ecological agroforestry system and natural regeneration.

Conventional agriculture included pumpkin (Cucurbita sp.), passion fruit (Passiflorine edulis) and oil palm (Bactris gasipaes) monocultures. In those areas, [10] also found 19 species of herbaceous plants, most of them belonging to Euphorbiaceae, La- miaceae and Poaceae. No arboreal species, except oil palm, were found.

Pastures were mainly composed by Brachiaria spp. and $C y$ perus meyenianus and were characterized by different disturbing degrees (according to the presence of termite colonies, indicators vegetation, soil erosion and information about cattle productivity). The vegetation of those areas included 27 species of herbaceous plants, mainly Fabaceae, Asteraceae and Poaceae, and one specimen of Psidium guajava tree [10].

Ecological-based agroforestry and natural regenerations differed from each other by their age, as an indirect measure of system development and succession. So, agroforestry replicates were 4-, 8- and 16-years old, while natural regenerations were 5-, 20- and 30-years old. Moreover, in those ecological agroforestry systems, [10] found 46 arboreal and 62 herbaceous species of 33 botanical families (mainly Fabaceae, Lauraceae, Arecaceae, Euphorbiaceae, Sapindaceae, Verbenaceae and Poaceae). Natural regenerations were mostly composed by 45 arboreal and 58 herbaceous species, belonging also to 33 families, with emphasis to Fabaceae, Lauraceae, Myrtaceae, Euphorbiaceae, Moraceae, Annonaceae, Arecaceae, Rubiaceae, Verbenaceae and Asteraceae [10].

Table 1: Physical and chemical attributes at arable layer of soils collected from conventional agriculture, ecological agroforestry, pasture and natural regenerations, and the organic compost used for external fertilization on conventional agriculture areas, every three months (100g plant 1). Lower cases indicate statistical significance (Tukey, $p<0.05$ ) among land-use system (LUS). nd - not determined. Source: Froufe et al. [10].

\begin{tabular}{|c|c|c|c|c|c|c|}
\hline Land-Use System & Porosity $\mathrm{Cm} \mathrm{Cm}^{-3}$ & Bulk Density Kg Dm ${ }^{-3}$ & $\mathrm{Ph}\left(\mathrm{Cacl}_{2}\right)$ & $\mathbf{A l}^{3+}$ & $\mathrm{H}^{+}+\mathrm{Al}^{3+}$ & M \\
\hline Conventional Agriculture & $0.6 \mathrm{a}$ & $1.1 \mathrm{~b}$ & $6.3 \mathrm{a}$ & $0.02 \mathrm{~b}$ & $3.2 \mathrm{~b}$ & $0.2 \mathrm{~b}$ \\
\hline Ecological Agroforestry & $0.6 \mathrm{a}$ & $1.1 \mathrm{~b}$ & $5.5 \mathrm{~b}$ & $0.12 \mathrm{~b}$ & $4.1 \mathrm{~b}$ & $3.1 \mathrm{~b}$ \\
\hline Pasture & $0.5 \mathrm{~b}$ & $1.2 \mathrm{a}$ & $5.4 \mathrm{~b}$ & $0.05 \mathrm{~b}$ & $3.8 \mathrm{~b}$ & $1.4 \mathrm{~b}$ \\
\hline Natural Regeneration & $0.5 \mathrm{~b}$ & $1.0 \mathrm{~b}$ & $4.5 \mathrm{c}$ & $0.78 \mathrm{a}$ & $6.8 \mathrm{a}$ & $21.4 \mathrm{a}$ \\
\hline \multirow[t]{2}{*}{ Organic Compost } & nd & nd & 8.3 & 0 & 1.6 & 2.4 \\
\hline & P mg.dm ${ }^{-3}$ & K cmol.dm ${ }^{-3}$ & Ca cmol.dm-3 & Mg cmol.dm-3 & \multicolumn{2}{|c|}{ V \% } \\
\hline \multirow{6}{*}{$\begin{array}{c}\text { Conventional Agriculture } \\
\text { Ecological Agroforestry } \\
\text { Pasture } \\
\text { Natural Regeneration } \\
\text { Organic Compost }\end{array}$} & $16.8 \mathrm{a}$ & 0.2 & $7.6 \mathrm{a}$ & $7.1 \mathrm{a}$ & \multicolumn{2}{|c|}{$81.6 \mathrm{a}$} \\
\hline & $11.0 \mathrm{~b}$ & 0.2 & $4.3 \mathrm{~b}$ & $3.0 \mathrm{~b}$ & \multicolumn{2}{|c|}{$62.9 \mathrm{a}$} \\
\hline & $10.6 \mathrm{~b}$ & 0.2 & $2.9 \mathrm{~b}$ & $2.2 \mathrm{~b}$ & \multicolumn{2}{|c|}{$55.0 \mathrm{a}$} \\
\hline & $10.6 \mathrm{~b}$ & 0.2 & $1.3 \mathrm{c}$ & $2.2 \mathrm{~b}$ & \multicolumn{2}{|c|}{$34.7 \mathrm{~b}$} \\
\hline & 966.3 & 12.2 & 4.2 & 3.3 & \multicolumn{2}{|c|}{44.1} \\
\hline & N\% & $\mathrm{COg} \mathrm{kg}^{-1}$ & $\begin{array}{l}\text { Arboreal Bio- } \\
\text { mass } \mathrm{Mg} \mathrm{ha}^{-1}\end{array}$ & $\begin{array}{l}\text { Herbaceous Biomass } \\
\qquad \mathrm{Mg} \mathrm{ha}^{-1}\end{array}$ & \multicolumn{2}{|c|}{ Soil Carbon $\mathrm{Mg} \mathrm{ha}^{-1}$} \\
\hline \multirow{2}{*}{$\begin{array}{l}\text { Conventional Agriculture } \\
\text { Ecological Agroforestry }\end{array}$} & $0.2 \mathrm{a}$ & $19.2 \mathrm{a}$ & $7.2 \mathrm{c}$ & 0.01 & \multicolumn{2}{|c|}{38.3} \\
\hline & $0.2 \mathrm{a}$ & $18.2 \mathrm{ab}$ & $32.4 \mathrm{~b}$ & 0.01 & \multicolumn{2}{|c|}{38.6} \\
\hline Pasture & $0.1 \mathrm{~b}$ & $15.6 \mathrm{~b}$ & 0 & 0.01 & \multicolumn{2}{|c|}{33.9} \\
\hline Natural Regeneration & $0.2 \mathrm{a}$ & $18.5 \mathrm{ab}$ & $70.8 \mathrm{a}$ & 0.01 & \multicolumn{2}{|c|}{39} \\
\hline Organic Compost & 1.2 & 142.5 & nd & nd & \multicolumn{2}{|c|}{ nd } \\
\hline
\end{tabular}

Three $25 \times 10 \mathrm{~m}$ plots were installed at small farm containing each land-use system (LUS), totalizing nine plots per LUS. The soil was predominantly characterized as Inceptisol and Entisol [10], which physical and chemical attributes are shown at Table 1.

\section{Standing litter biomass and nutrient contents}

Standing little was collected directly from the soil, on all 36 plots, by randomized triplicate samplings. Standing litter was evaluated from July 2007 - March 2008. All 108 samples were gently washed to remove soil particles, dried $\left(60^{\circ} \mathrm{C}\right)$ and separated into: 
a) leaves;

b) branches;

c) reproductive structures (flowers, fruits and seeds);

d) barks; and

e) fine roots and weighted in a dry basis $\left(\mathrm{g} \mathrm{m}^{-2}\right.$, transformed to $\mathrm{Mgha}^{-1}$ afterwards).

Litter fractions were summed to determine the total amount of standing litter accumulated aboveground. Replicates of litter fractions were grounded, sieved and analysed for their nutrient

Results contents. Nitrogen was determined by Kjeldahl method; while P, $\mathrm{K}, \mathrm{Ca}$ and $\mathrm{Mg}$ were evaluated by dry digestion followed by atomic absorption ( $\mathrm{Ca}$ and $\mathrm{Mg}$ ), emission spectrophotometry (K) and colorimetric spectrophotometry (P).

\section{Statistical analysis}

All data were analysed as randomly block (three replicates), and ANOVA and Tukey tests were performed with SPSS statistical package (SPSS, 1991). Because of their heterogeneity and to improve conformity to normal distributions, reproductive structures and barks data were transformed $(y=\sqrt{x+1,0})$ prior to statistical analysis.

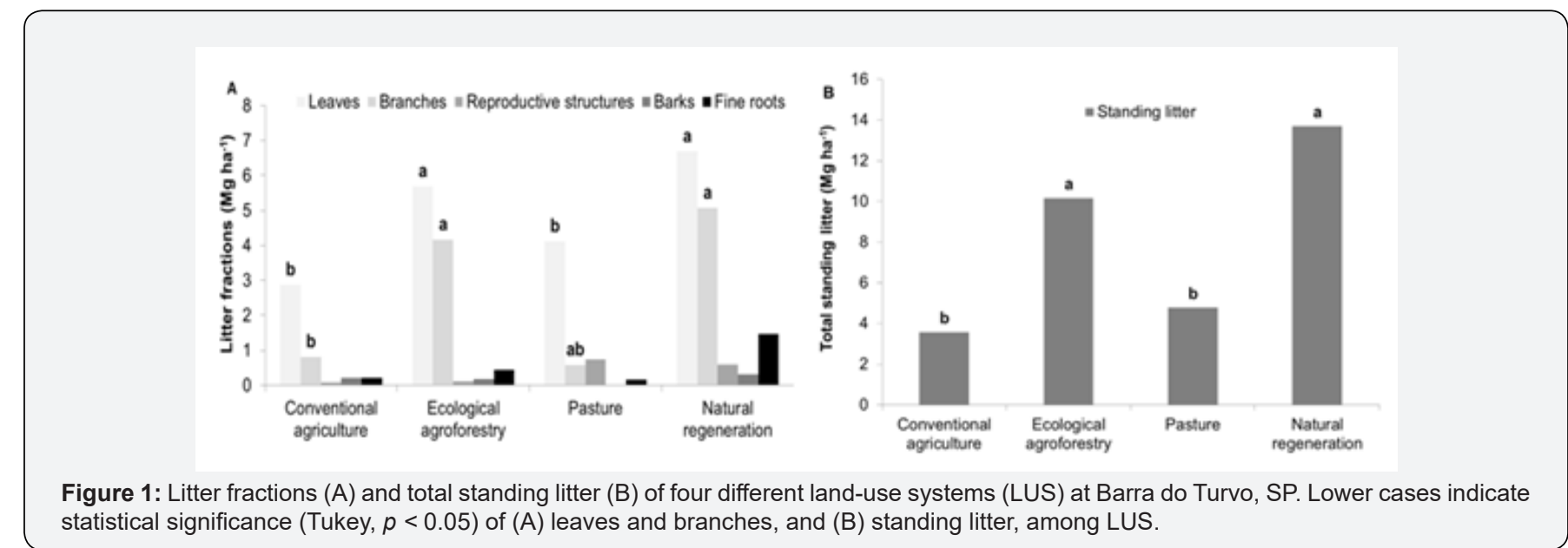

Table 2: Capital letters indicate statistical significance (Tukey, $p<0.05$ ) among litter fractions of each land-use system (LUS); while lower cases indicate statistical significance of LUS for each litter fraction. Relative composition of standing litter from different agricultural and natural landuse systems at Barra do Turvo, SP.

\begin{tabular}{|c|c|c|c|c|c|}
\hline & $\begin{array}{c}\text { Leaves \% of Total } \\
\text { Standing Litter }\end{array}$ & $\begin{array}{c}\text { Branches \% of Total } \\
\text { Standing Litter }\end{array}$ & $\begin{array}{c}\text { Reproductive Strut\% of } \\
\text { Total Standing Literatures }\end{array}$ & $\begin{array}{c}\text { Barks \% of Total } \\
\text { Standing Litter }\end{array}$ & $\begin{array}{c}\text { Fine Roots \% of To- } \\
\text { tal Standing Litter }\end{array}$ \\
\hline Conventional Agriculture & $80.0 \mathrm{~A} \mathrm{a}$ & $22.6 \mathrm{~B} \mathrm{bc}$ & $2.5 \mathrm{~B}$ & $5.8 \mathrm{~B}$ & $6.1 \mathrm{~B}$ \\
\hline Ecological Agroforestry & $55.9 \mathrm{~A} \mathrm{~b}$ & $40.9 \mathrm{~A} \mathrm{a}$ & $1.2 \mathrm{~B}$ & $1.7 \mathrm{~B}$ & $4.4 \mathrm{~B}$ \\
\hline Pasture & $85.8 \mathrm{~A} \mathrm{a}$ & $12.1 \mathrm{~B} \mathrm{c}$ & $15.4 \mathrm{~B}$ & $0.2 \mathrm{~B}$ & $3.4 \mathrm{~B}$ \\
\hline Natural Regeneration & $48.7 \mathrm{~A} \mathrm{~b}$ & $36.9 \mathrm{~B} \mathrm{ab}$ & $4.4 \mathrm{C}$ & $2.3 \mathrm{C}$ & $10.7 \mathrm{C}$ \\
\hline
\end{tabular}

Standing litter was significantly greater in diverse systems (natural regeneration and ecological agroforestry) than monocultures (conventional agriculture and pasture), varying from 3.6 to $13.7 \mathrm{Mg} \mathrm{ha}^{-1}$ (Figure 1), and was mostly composed, among all land-use systems, by leaves (66.1\%) and branches (29.2\%). The other litter fractions greatly varied among LUS, and represented only $7.2 \%$ (fine roots), $4.3 \%$ (reproductive structures) and $2.1 \%$ (barks). Regarding each LUS, diverse systems also generate a more diverse litter (Table 2), while monocultures standing litter were predominantly formed by leaves.

Regarding the entire data set, conventional agriculture generated a richer standing litter on nitrogen, phosphorous, calcium and magnesium as compared to ecological agroforestry and natural regenerations, while pastures produced a poorer standing litter for all nutrients, except potassium (Table 3). Additionally, leaf litter was richer in all nutrients, while branches, barks and fine roots had the lowest nutrient contents.

Litter fractions of conventional agriculture had highest nutrient content values than ecological agroforestry, natural regenerations and pasture (Table 4). As a result of biomass production and chemical quality, the total amount of nutrients returned to soil system from standing litter varied from $11.5 \mathrm{~kg} \mathrm{ha}^{-1}(\mathrm{Mg})$ to $346.9 \mathrm{~kg} \mathrm{ha}^{-1}(\mathrm{~K})$.

Despite their higher efficiency on nutrient use (Table 5), lower amounts of nutrients returned to soil systems from standing litter of pastures and conventional agriculture, while standing litter from natural regeneration was responsible for greater amounts of $\mathrm{N}, \mathrm{P}, \mathrm{Ca}$ and Mg return, and greater amounts of $\mathrm{K}$ were observed from standing litter of ecological agroforestry (Figure 2). 
Table 3: Nutrient contents $\left(\mathrm{g} \mathrm{kg}^{-1}\right)$ of all standing litter (upper data) and litter fractions (bottom data) collected from different agricultural agrosystems and natural regenerations, at Barra do Turvo, SP.

\begin{tabular}{|c|c|c|c|c|c|}
\hline Land-Use System & $\mathrm{N}$ g.kg ${ }^{-1}\left(=\mathrm{kg} \cdot \mathrm{Mg}^{-1}\right)$ & g.kg-1 (= kg. $\left.\mathrm{Mg}^{-1}\right)$ & $\mathrm{Kg} \cdot \mathrm{kg}^{-1}\left(=\mathrm{kg} \cdot \mathrm{Mg}^{-1}\right)$ & Ca g.kg ${ }^{-1}\left(=\mathrm{kg} \cdot \mathrm{Mg}^{-1}\right)$ & ${\mathrm{Mg} \mathrm{g} \cdot \mathrm{kg}^{-1}}\left(=\mathrm{kg} \cdot \mathrm{Mg}^{-1}\right)$ \\
\hline $\begin{array}{l}\text { Conventional Agri- } \\
\text { culture }\end{array}$ & $13.3 \mathrm{~A}$ & $4.3 \mathrm{~A}$ & $12.5 \mathrm{~B}$ & $13.2 \mathrm{~A}$ & $5.2 \mathrm{~A}$ \\
\hline $\begin{array}{l}\text { Ecological Agrofor- } \\
\text { estry }\end{array}$ & $11.7 \mathrm{~B}$ & $2.9 \mathrm{~B}$ & $17.8 \mathrm{AB}$ & $13.6 \mathrm{~A}$ & $2.9 \mathrm{~B}$ \\
\hline Pasture & $9.8 \mathrm{C}$ & $2.9 \mathrm{~B}$ & $22.3 \mathrm{~A}$ & $7.7 \mathrm{~B}$ & $2.2 \mathrm{C}$ \\
\hline $\begin{array}{c}\text { Natural Regener- } \\
\text { ation }\end{array}$ & $11.3 \mathrm{~B}$ & $2.5 \mathrm{~B}$ & $14.0 \mathrm{~B}$ & $12.0 \mathrm{~A}$ & $3.0 \mathrm{~B}$ \\
\hline Litter Fractions & $\mathrm{Ng} \cdot \mathrm{kg}^{-1}\left(=\mathrm{kg} \cdot \mathrm{Mg}^{-1}\right)$ & P g.kg ${ }^{-1}\left(=\right.$ kg. $\left.\mathrm{Mg}^{-1}\right)$ & $\mathrm{Kg} \cdot \mathrm{kg}^{-1}\left(=\mathrm{kg} \cdot \mathrm{Mg}^{-1}\right)$ & $\mathrm{Ca}$ & ${\mathrm{Mg} \mathrm{g} \cdot \mathrm{kg}^{-1}}\left(=\mathrm{kg} \cdot \mathrm{Mg}^{-1}\right)$ \\
\hline Leaves & $15.8 \mathrm{a}$ & $3.9 \mathrm{a}$ & $39.7 \mathrm{a}$ & $14.5 \mathrm{ab}$ & $3.9 \mathrm{a}$ \\
\hline Branches & $7.5 \mathrm{~d}$ & $2.2 \mathrm{c}$ & $4.4 \mathrm{~b}$ & $11.3 \mathrm{bc}$ & $2.9 \mathrm{bc}$ \\
\hline $\begin{array}{l}\text { Reproductive } \\
\text { Structures }\end{array}$ & $12.6 \mathrm{~b}$ & $2.7 \mathrm{bc}$ & $6.9 \mathrm{~b}$ & $10.1 \mathrm{c}$ & $2.3 \mathrm{c}$ \\
\hline Barks & $10.1 \mathrm{c}$ & $2.4 \mathrm{c}$ & $2.5 \mathrm{~b}$ & $15.0 \mathrm{a}$ & $2.8 \mathrm{bc}$ \\
\hline Fine Roots & $10.0 \mathrm{c}$ & $3.2 \mathrm{~b}$ & $8.2 \mathrm{~b}$ & $6.4 \mathrm{~d}$ & $3.1 \mathrm{~b}$ \\
\hline
\end{tabular}

Table 4: Lower cases indicate statistical significance (Tukey, $p<0.05$ ) of litter fractions amounts and nutrient contents among land-use system (LUS). Nutrient contents $\left(\mathrm{g} \mathrm{kg}^{-1}\right)$ and ratios of litter fractions of standing litter collected from different agricultural agrosystems and natural regenerations, at Barra do Turvo, SP.

\begin{tabular}{|c|c|c|c|c|c|c|c|}
\hline Land-Use System & $\mathbf{N}$ & $\begin{array}{c}\text { Pg.kg-1 (= kg. } \\
\text { Mg-1) }\end{array}$ & $\begin{array}{c}\text { Kg.kg-1 (= kg. } \\
\text { Mg-1) }\end{array}$ & Ca g.kg-1 (= kg. Mg-1) & Mgg.kg-1 (= kg. Mg-1) & CN & NP \\
\hline \multicolumn{8}{|c|}{ Leaves } \\
\hline Conventional Agriculture & 18.7 a & $6.5 \mathrm{a}$ & $16.5 \mathrm{~b}$ & $17.2 \mathrm{a}$ & $6.8 \mathrm{a}$ & $25.2 \mathrm{~b}$ & $3.2 \mathrm{~b}$ \\
\hline Ecological Agroforestry & $17.0 \mathrm{a}$ & $3.0 \mathrm{~b}$ & $46.8 \mathrm{a}$ & $16.7 \mathrm{a}$ & $3.4 \mathrm{~b}$ & $27.8 \mathrm{~b}$ & $6.6 \mathrm{a}$ \\
\hline Pasture & $10.2 \mathrm{~b}$ & $3.5 \mathrm{~b}$ & $54.4 \mathrm{a}$ & $8.6 \mathrm{~b}$ & $1.8 \mathrm{c}$ & $46.5 \mathrm{a}$ & $3.4 \mathrm{~b}$ \\
\hline Natural Regeneration & $17.3 \mathrm{a}$ & $2.6 \mathrm{~b}$ & $41.9 \mathrm{a}$ & $15.4 \mathrm{a}$ & $3.4 \mathrm{~b}$ & $27.1 \mathrm{~b}$ & $7.3 \mathrm{a}$ \\
\hline \multicolumn{8}{|c|}{ Branches } \\
\hline Conventional Agriculture & $8.3 \mathrm{a}$ & $1.5 \mathrm{~b}$ & $7.9 \mathrm{a}$ & $11.2 \mathrm{ab}$ & $4.1 \mathrm{a}$ & 54.9 & $6.9 \mathrm{ab}$ \\
\hline Ecological Agroforestry & $7.5 \mathrm{ab}$ & $1.6 \mathrm{~b}$ & $3.3 \mathrm{~b}$ & $11.1 \mathrm{ab}$ & $2.1 \mathrm{~b}$ & 62 & $9.2 \mathrm{a}$ \\
\hline Pasture & $7.4 \mathrm{ab}$ & $3.0 \mathrm{a}$ & $4.0 \mathrm{~b}$ & $9.2 \mathrm{~b}$ & $2.7 \mathrm{~b}$ & 58.3 & $2.2 \mathrm{~b}$ \\
\hline Natural Regeneration & $7.2 \mathrm{~b}$ & $2.6 \mathrm{a}$ & $3.0 \mathrm{~b}$ & $13.4 \mathrm{a}$ & $2.8 \mathrm{~b}$ & 64.4 & $2.8 \mathrm{~b}$ \\
\hline \multicolumn{8}{|c|}{ Reproductive Structures } \\
\hline Conventional Agriculture & $12.4 \mathrm{a}$ & $4.8 \mathrm{a}$ & 13.1 & 3.4 & $2.2 \mathrm{~b}$ & $36.5 \mathrm{a}$ & $2.7 \mathrm{~b}$ \\
\hline Ecological Agroforestry & $12.0 \mathrm{a}$ & $3.3 \mathrm{ab}$ & 7.2 & 17 & $2.6 \mathrm{~b}$ & $37.7 \mathrm{a}$ & $3.9 \mathrm{~b}$ \\
\hline Pasture & $14.8 \mathrm{~b}$ & $1.7 \mathrm{~b}$ & 5.1 & 17.4 & $4.3 \mathrm{a}$ & $30.4 \mathrm{~b}$ & $8.6 \mathrm{a}$ \\
\hline Natural Regeneration & $12.7 \mathrm{a}$ & $2.3 \mathrm{~b}$ & 6.3 & 6.5 & $1.9 \mathrm{~b}$ & $35.3 \mathrm{~b}$ & $6.1 \mathrm{ab}$ \\
\hline \multicolumn{8}{|c|}{ Barks } \\
\hline Conventional Agriculture & $8.6 \mathrm{~b}$ & $3.6 \mathrm{a}$ & $5.3 \mathrm{a}$ & $8.4 \mathrm{ab}$ & 3.3 & 52.8 & 2.4 \\
\hline Ecological Agroforestry & $11.6 \mathrm{a}$ & $2.8 \mathrm{ab}$ & $2.6 \mathrm{ab}$ & $14.9 \mathrm{ab}$ & 2.9 & 38.8 & 5 \\
\hline Pasture & $10.5 \mathrm{ab}$ & $1.8 \mathrm{ab}$ & $2.2 \mathrm{ab}$ & $6.0 \mathrm{~b}$ & 1.9 & 43.1 & 5.7 \\
\hline Natural Regeneration & $8.7 \mathrm{~b}$ & $1.6 \mathrm{~b}$ & $1.8 \mathrm{~b}$ & $18.2 \mathrm{a}$ & 2.6 & 56 & 5.7 \\
\hline \multicolumn{8}{|c|}{ Fine Roots } \\
\hline Conventional Agriculture & $8.8 \mathrm{~b}$ & $4.3 \mathrm{ab}$ & $14.5 \mathrm{a}$ & 6.6 & $3.1 \mathrm{ab}$ & 51.4 & $2.2 \mathrm{~b}$ \\
\hline Ecological Agroforestry & $8.8 \mathrm{~b}$ & $5.2 \mathrm{a}$ & $12.0 \mathrm{a}$ & 7.4 & $3.8 \mathrm{a}$ & 52.1 & $2.4 \mathrm{~b}$ \\
\hline Pasture & $11.9 \mathrm{a}$ & $2.3 \mathrm{c}$ & $4.6 \mathrm{~b}$ & 3.8 & $2.0 \mathrm{~b}$ & 40.5 & $5.5 \mathrm{a}$ \\
\hline Natural Regeneration & $9.4 \mathrm{ab}$ & $2.7 \mathrm{bc}$ & $8.0 \mathrm{ab}$ & 7.9 & $3.7 \mathrm{a}$ & 50.4 & $3.7 \mathrm{~b}$ \\
\hline
\end{tabular}


JOJ Horticulture \& Arboriculture

Table 5: Lower cases indicate statistical significance (Tukey, $p<0.05$ ) among land-use systems.

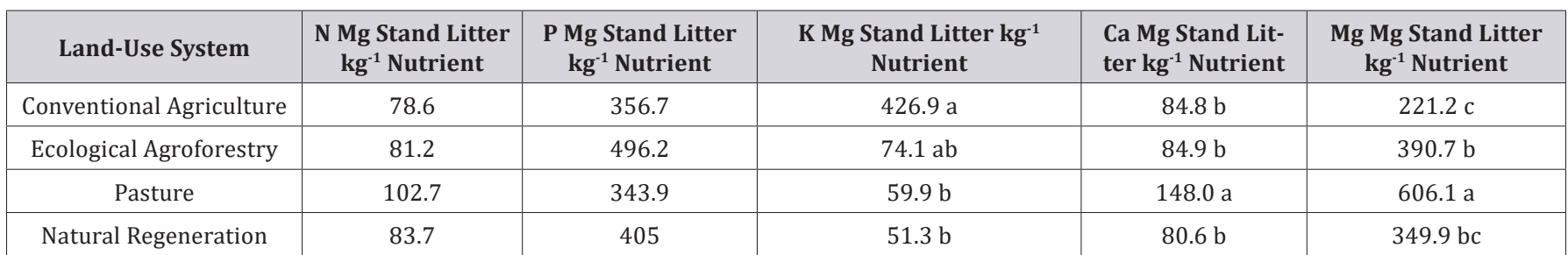

Nutrient use efficiency for standing litter production of different agricultural agrosystems and natural regenerations, at Barra do Turvo, SP.

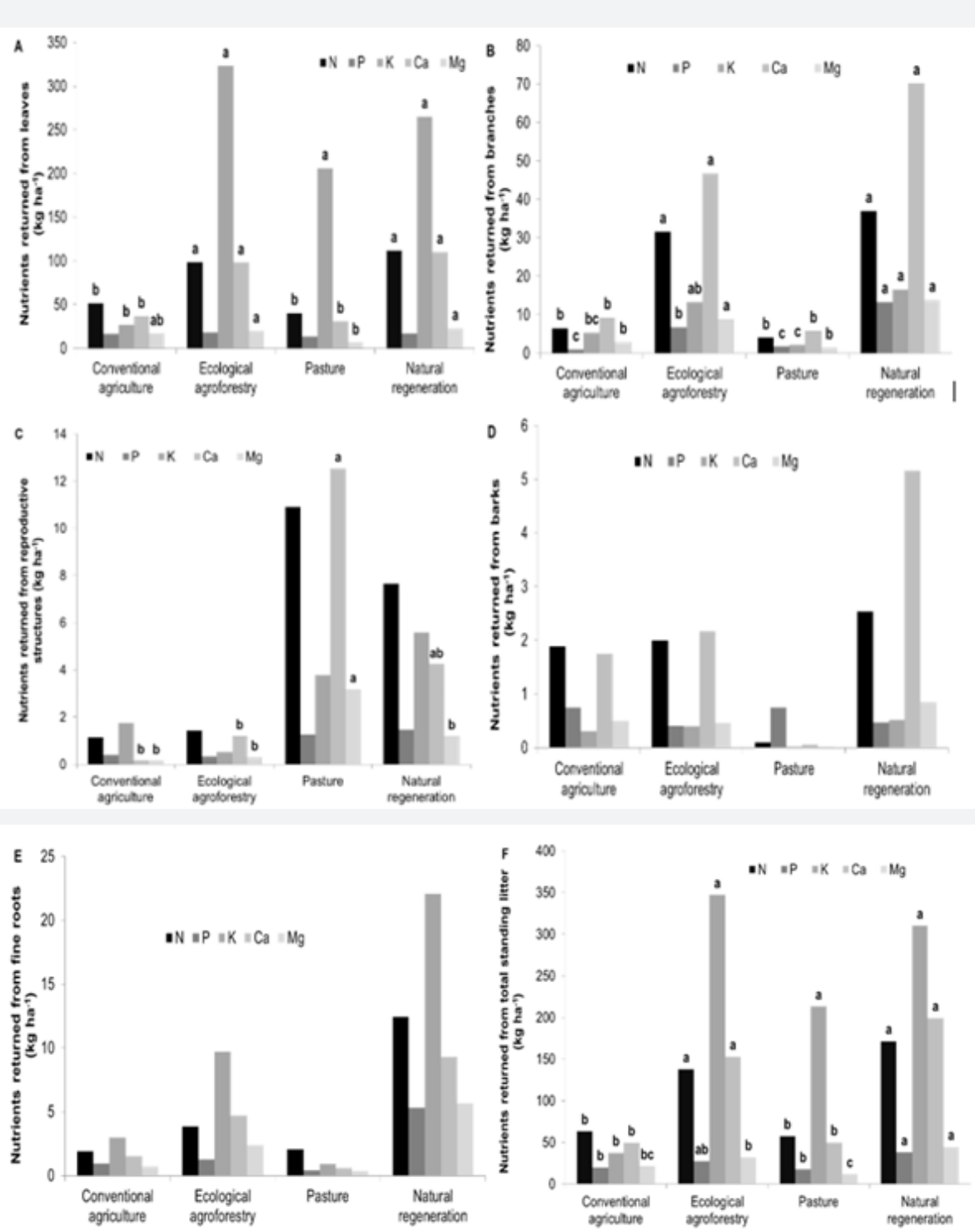

Figure 2: Nutrients ( $\mathrm{N}, \mathrm{P}, \mathrm{K}, \mathrm{Ca}, \mathrm{Mg}$ ) returned to soil ( $\mathrm{kg}$ ha-1) from litter fractions and total standing litter of four different landuse systems (LUS) at Barra do Turvo, SP. Lower cases indicate statistical significance (Tukey, $p<0.05$ ) for each nutrient, among LUS. (A) leaves; (B) branches; (C) reproductive structures; (D) barks; (E) fine roots; $(F)$ total standing litter.

\section{Discussion}

Standing litter amounts of ecological agroforestry and natural regeneration were strongly lower than previous results on similar land-use systems [13]. Litter production is a result of age and vegetation diversity [16] and leaves usually represent over than $60 \%$ of litter fractions on natural and agroforestry systems [17-19].
Ecological agroforestry and natural forests, because of their higher diversity, generate a diverse litter layer, where soil macrofauna community and animal activity contribute to enhance litter decomposition, biogeochemical cycles and the long-term functioning of ecosystems [13,20-23] By the other hand, herbaceous dominated landscapes have low potential to mitigate carbon losses from agroecosystems and need strong management strategies toward to sustainability [24]. 
The great production of standing litter on ecological agroforestry can be a response of intensive pruning management. Those ecological agroforestry systems usually reach their maximum production until 8 years old [25]. After that age, the agricultural management is usually stopped, because of massive arboreal development and legal restrictions to forest management, thus those areas turn to natural regenerations.

Under forestry and agricultural management, biomass removals of logs, fruits and seeds imply a great loss of soil nutrients and the reduction of soil fertility [26,27] Despite agricultural and timber production, pruning management and the maintenance of foliar structures on soil appear to represent a boundary line towards to the sustainability of ecological agroforestry [28-31].

According to our results and the nutrient use efficiency, proposed by [32]. as an index to define better strategies to produce biomass in response to nutrient availability, it could be expectable that pastures and conventional agriculture would be more efficient on biomass production. Despite this, and because pastures were mainly composed by Cuphea racemosa, Desmodium adscendens, Oxalis corymbosa, Panicum maximum, Solidago chilensis, Thelypteris dentate, Vernonia polyanthes and Waltheria indica [10], all acid soil resistant and/or invasive plants $[33,34]$, those pastures advance toward a high stressed level of unsustainable management. Still, conventional agriculture productivity is guaranteed only because of the addition of external mineral and organic fertilization [10] being naturally unsustainable at long time.

For ecological agroforestry, characterized by no exogenous inputs used to maintain its productivity, midterm NUE values were similar to those of natural regenerations. Along with its pruning management linked to biomass production and decomposition, ecological agroforestry sustainability thus resembles to natural forest areas.

Despite all results indicating the sustainability of the ecological-based agroforestry here evaluated, it cannot be considered as a simple solution to environmental and/or pollution mitigation. An optimal strategy for these purposes depends on a wide range of technical criteria, also including yield production, economical returns to farmers, environmental issues, current and future scenarios and trade-offs among all these variables [35] However, ecological-based agroforestry systems appear to positively contribute to expand the range of possibilities of small farmers on food and wood production [36] and also regulate ecosystems functions in agricultural and boundary landscapes [24,37], by carbon and nutrient additions to soil from litter structures [3842].

\section{Conclusion}

Species diversity land-use systems imply greater amounts of stand litter than monocultures. Similar patterns are observed for nutrients returned to soil from standing litter. Ecological-based agroforestry systems is an environmentally friendly land-use system, regarding to their sustainability and have elevated potential to recover soil fertility in agroecosystems.

\section{Acknowledgement}

The author would like to thank to Integral Technical Assistance Coordination (CATI, SP) and Association of Agroforestry Farmers of Barra do Turvo and Adrianopolis (Cooperafloresta), for family small farm indications, and technical and logistical support. Capital letters indicate statistical significance (Tukey, $p$ $<0.05$ ) of litter fractions amounts and nutrient contents among land-use system (LUS). Lower cases indicate statistical significance (Tukey, $\mathrm{p}<0.05$ ) of litter fractions amounts and nutrient contents among land-use system (LUS).

\section{References}

1. Tabarelli M, Lopes AV, Peres CA (2008) Edge-effects drive tropical forest fragments towards an early-successional system. Biotropica 40(6): 657-661.

2. Ribeiro MC, Metzger JP, Martensen AC, Ponzoni FJ, Hirota MM (2009) The Brazilian Atlantic Forest: How much is left, and how is the remaining forest distributed? Implications for conservation Biological Conservation. 142(6): 1141-1153

3. Murty D, Kirschbaum MUF, Mc Murtrie RE, Mc Gilvray H (2002) Does conversion of forest to agricultural land change soil carbon and nitrogen? A review of the literature. Global Change Biology 8: 105-123.

4. Marin-Spiotta E, Silver WL, Swanston CW, Ostertag R (2009) Soil organic matter dynamics during 80 years of reforestation of tropical pastures. Global Change Biology 15(6): 1584-1597.

5. Don A, Schumacher J, Freibauer A (2011) Impact of tropical land-use change on soil organic carbon stocks - a meta-analysis. Global Change Biology 17: 1658-1670.

6. Miranda E, Carom J, Couto E, Camargo P (2016) Long-term changes in carbon soil stocks in the Brazilian Cerrado under scommercial soybean. Land Degradation \& Development 27(6): 1586-1594.

7. Hector A, Beale AJ, Minns A, Otway SJ, Lawton JH (2000) Consequences of the reduction of plant diversity for litter decomposition: effects through litter quality and microenvironment. Oikos 90: 357-371.

8. Hättenschwiler S, Gasser P (2005) Soil animals alter plant litter diversity effects on decomposition. PNAS 102(5): 1519-1524.

9. Scherr SJ, Buck L, Willemen L, Milder JC (2014) Ecoagriculture: integrated landscape management for people, food, and nature. Encyclopedia of Agriculture and Food Systems (3): 1-17.

10. Froufe LCM, Seoane CES (2011) Levantamento fitossociológico comparativo entre sistema agroflorestal multiestrato e capoeiras como ferramenta para a execução da reserva legal. Pesquisa Florestal Brasileira, Colombo, 31(67): 203-225.

11. Das T, Das AK (2010) Litter production and decomposition in the forested areas of traditional homegardens: a case study from Barak Valley, Assam, northeast India. Agroforest Systems 79: 157-170.

12. Cezar RM, Vezzani FM, Schwiderke DK, Gaiad S, Brown GG, et al. (2015) Soil biological properties in multistrata successional agroforestry systems and in natural regeneration. Agroforest Systems 89: 1035-1047.

13. Froufe LCM, Schwiderke DK, Castellano AC, Cezar RM, Steenbock W, et al. (2019) Nutrient cycling from leaf litter in multistrata successional agroforestry systems and natural regeneration at Brazilian Atlantic Rainforest Biome. Agroforest Syst, pp. 1-13.

14. Veloso HP, Góes-Filho L (1982) Fitogeografia brasileira: classificação 
fisionômico-ecológica da vegetação neotropical. Salvador, BA: [s.n.], p 85, Projeto RADAMBRASIL.

15. Alvares CA, Stape JL, Sentelhas PC, Goncalves JLM, Sparovek G (2013) Köppen's climate classification map for Brazil. Meteorol Z 22(6): 1-18.

16. Shanin V, Valkonen S, Grabarnik P, Mäkipää R (2016) Using forest ecosystem simulation model EFIMOD in planning uneven-aged forest management. Forest Ecology and Management (378): 193-205.

17. Das DK, Chaturvedi OP (2005) Structure and function of Populus deltoides agroforestry systems in eastern India: 1. Dry matter dynamics. Agroforestry System 65: 215-221.

18. Isaac RS, Nair MA (2006) Litter dynamics of six multipurpose trees in a home garden in Southern Kerala, India. Agroforestry Systems 67: 203213.

19. Das T, Das AK (2010) Litter production and decomposition in the forested areas of traditional homegardens: a case study from Barak Valley, Assam, northeast India. Agroforest Systems 79: 157-170.

20. Zeng D, Mao R, Chang CSX, Li J, Yang D (2010) Carbon mineralization of tree leaf litter and crop residues from poplar-based agroforestry systems in Northeast China: A laboratory study. Applied Soil Ecology 44(2): 133-137.

21. Cezar RM, Vezzani FM, Schwiderke DK, Gaiad S, Brown GG, et al. (2015) Soil biological properties in multistrata successional agroforestry systems and in natural regeneration. Agroforest Systems 89: 1035-1047.

22. Li S, Tong Y, Wang Z (2017) Species and genetic diversity affect leaf litter decomposition in subtropical broadleaved forest in southern China. Journal of Plant Ecology 10(1): 232-241.

23. Santonja M, Rancon A, Fromin N, Baldy V, Hättenschwiler S, et al (2017) Plant litter diversity increases microbial abundance, fungal diversity, and carbon and nitrogen cycling in a Mediterranean shrubland. Soil Biology \& Biochemistry 111: 124-134.

24. D’Acunto L Semmartin M, Ghersa CM (2014) Uncropped fields margins to mitigate soil carbon losses in agricultural landscapes. Agriculture, Ecosystems and Environment 183: 60-68.

25. Frouf LCM, Rachwal MFG, Seoane CES ( 2011) Potencial de sistemas agroflorestais multiestrata para sequestro de carbono em áreas de ocorrência de Floresta Atlântica. Pesquisa Florestal Brasileira, Colombo, 31(66): 143-154

26. Malagoli P, Laine P, Rossato L, Ourry A (2005) Dynamics of nitrogen uptake and mobilization in field-grown winter oilseed rape (Brassica napus) from stem extension to harvest. Ann Bot 95(7): 853-861.

27. Masclaux-Daubresse C, Daniel-Vedele F, Dechorgnat J, Chardon F, Gaufichon L, et al. (2010) Nitrogen uptake, assimilation and remobilization in plants: challenges for sustainable and productive agriculture. Annals of Botany 105(7): 1141-1157.

28. Kumar BM (2011) Species richness and aboveground carbon stocks in the homegardens of central Kerala, India. Agriculture Ecosystems and Environment 140: 430-440.

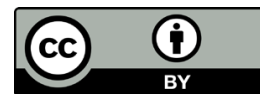

This work is licensed under Creative

Commons Attribution 4.0 License

DOI: 10.19080/JOJHA.2018.01.555592
29. Carnol M, Bazgir M (2013) Nutrient return to the forest floor through litter and throughfall under 7 forest species after conversion from Norway spruce. Forest Ecology and Management 309: 69-75.

30. Noponen MRA, Healey JR, Soto G, Haggar JP (2013) Sink or source The potential of coffee agroforestry systems to sequester atmospheric $\mathrm{CO}_{2}$ into soil organic carbon. Agriculture, Ecosystems and Environment 175: 60-68.

31. Utomo B, Prawoto AA, Bonnet S, Bangviwat A., Gheewala SH (2016) Environmental performance of cocoa production from monoculture and agroforestry systems in Indonesia. Journal of Cleaner Production 134: 583-591.

32. Vitousek PM (1982) Nutrient cycling and nutrient use efficiency. American Naturalist 119: 553-572.

33. Biondi D, Pedrosa-Macedo JH (2008) Plantas invasoras encontradas na área urbana de Curitiba (PR). Floresta 38(1): 129-144.

34. Valadares RT, Souza FBC, Castro NGD, Peres ALSS, Schneider SZ, et al. (2011) Levantamento florístico de um brejo-herbáceo localizado na restinga de Morada do Sol, município de Vila Velha, Espírito Santo, Brasil. Rodriguésia 62(4): 827-834.

35. Wade ASI, Asase A, Hadley P, Mason J, Ofori-Frimpong K, et al. (2010) Management strategies for maximizing carbon storage and tree species diversity in cocoa-growing landscapes. Agriculture, Ecosystems and Environment 138: 324-334.

36. Rice RA (2008) Agricultural intensification within agroforestry: the case of coffee and wood products. Agriculture, Ecosystems and Environment 128: 212-218.

37. Carsan S, Stroebel A, Dawson I, Kindt R, Mbow C, et al. (2014) Can agroforestry option values improve the functioning of drivers of agricultural intensification in Africa? Current Opinion in Environmental Sustainability $6: 35-40$

38. Béliveau A, Lucotte M, Davidson R, Paquet S, Mertens F, et al. (2017) Reduction of soil erosion and mercury losses in agroforestry systems compared to forests and cultivated fields in the Brazilian Amazon. Journal of Environmental Management 203: 522-532.

39. Chen C, Liu W, Jiang X, Wu J (2017) Effects of rubber-based agroforestry systems on soil aggregation and associated soil organic carbon: Implication for land use. Geoderma 299: 13-24.

40. Pardon P, Reubens B, Reheul D, Mertens J, de Frenne P, et al. (2017) Trees increase soil organic carbon and nutrient availability in temperate agroforestry systems. Agriculture, Ecosystems and Environment 247: 98-111.

41. SPSS (1991) SPSS statistical algorithms (2 $2^{\text {a }}$ edn). Chicago, IL: SPSS Inc.

42. Valadares RT, Souza FBC, Castro, NGD, Peres ALSS, Schneider SZ, et al. (2011) Levantamento florístico de um brejo-herbáceo localizado na restinga de Morada do Sol, município de Vila Velha, Espírito Santo, Brasil. Rodriguésia 62(4): 827-834.

\section{Your next submission with Juniper Publishers} will reach you the below assets

- Quality Editorial service

- Swift Peer Review

- Reprints availability

- E-prints Service

- Manuscript Podcast for convenient understanding

- Global attainment for your research

- Manuscript accessibility in different formats

( Pdf, E-pub, Full Text, Audio)

- Unceasing customer service

Track the below URL for one-step submission https://juniperpublishers.com/online-submission.php 